Proceedings of the Edinburgh Mathematical Society (2003) 46, 315-328 (C)

DOI:10.1017/S0013091502000548 Printed in the United Kingdom

\title{
A SHOCK LAYER IN PARABOLIC PERTURBATIONS OF A SCALAR CONSERVATION LAW
}

\author{
VLADIMIR SHELUKHIN \\ Lavrentyev Institute of Hydrodynamics, Novosibirsk, \\ 630090, Russia (shelukhin@hydro.nsc.ru)
}

(Received 23 May 2002)

\begin{abstract}
A definition of a shock layer of thickness $\delta$ is proposed when a parabolic perturbation is applied to a scalar conservation law. The asymptotic equality $\delta \asymp \sqrt{\varepsilon}$ is established, where $\varepsilon$ denotes viscosity. This equality is proved to be optimal. Nevertheless, the equality $\delta \asymp \varepsilon$ is also proved to be valid for a class of shocks in accordance with the Mises conjecture.
\end{abstract}

Keywords: Riemann problem; shock; parabolic perturbation

2000 Mathematics subject classification: Primary 35L67

Secondary $35 \mathrm{~K} 65$

\section{Definition of a shock layer}

Physically, shock wavefronts in gases are not surfaces but thin layers where the processes of internal friction, heat conductivity, chemical kinetics and molecular diffusion cannot be neglected. These processes are related to the size and structure of a shock layer [6]. In view of the complexity of an exhaustive description, it is customary to consider simplified models, where one aspect or another of the system is emphasized. Here we are concerned with the question of dependence between shock-layer thickness and viscosity.

We recall the conjecture of Mises [10] about the asymptotic behaviour of solutions $u^{\mu}$ of the viscous-gas equations as the viscosity $\mu$ goes to zero. Given a solution $u^{0}$ of the inviscid gas dynamics equations with a shock curve $\Gamma$, he supposed that there is a distance $d(\mu)$ from $\Gamma$, satisfying the condition $\mu / d(\mu) \rightarrow 0$, such that $u^{\mu}$ is close to $u^{0}$ at distances greater than $d$ from $\Gamma$, and $u^{\mu}$ is close to some shock structure profile at distances from $\Gamma$ of less than $d$. In what follows, we partly justify the Mises conjecture for a scalar conservation law.

We consider the Riemann problem

$$
U_{t}+f(U)_{x}=0,\left.\quad U\right|_{t=0}=u_{0}(x),
$$

with the initial data

$$
u_{0}(x)= \begin{cases}u_{+}, & x>0 \\ u_{-}, & x<0\end{cases}
$$


Given a flux function $f(u)$, a shock-wave solution occurs for a wide range of the initial constant states $u_{+}$and $u_{-}$. In particular, the function

$$
U(t, x)= \begin{cases}u_{-}, & x<\lambda t, \\ u_{+}, & x>\lambda t\end{cases}
$$

is a shock solution if

$$
u_{-}>u_{+} \quad \text { and } \quad f(u)=\lambda u+\varphi(u-\beta), \quad \beta=\frac{1}{2}\left(u_{-}+u_{+}\right),
$$

where $\lambda=$ const. and the function $\varphi(u)$ meets the restrictions

$$
0 \leqslant \varphi^{\prime \prime}(u) \in C^{2}(\mathbb{R}), \quad \varphi(-u)=\varphi(u) \quad \forall u \in R .
$$

Indeed, if

$$
\Gamma=\left\{(t, x): x=x_{0}(t), 0<t<T, x_{0}(0)=0\right\}
$$

is a discontinuity curve starting from the origin, then, by the Rankine-Hugoniot condition $[\mathbf{1 3}]$,

$$
x_{0}^{\prime}(t)=\frac{[f(u)]}{[u]}=\lambda+\frac{[\varphi(u-\beta)]}{[u]},
$$

where

$$
[u]=\lim _{\alpha \rightarrow 0}\left(u\left(t, x_{0}(t)+\alpha\right)-u\left(t, x_{0}(t)-\alpha\right)\right) .
$$

With $\varphi(u)$ being even, the function $\varphi(u-\beta)$ suffers no jump at the discontinuity curve $\Gamma$ :

$$
\varphi\left(u_{+}-\beta\right)-\varphi\left(u_{-}-\beta\right)=\varphi\left(\frac{1}{2}\left(u_{+}-u_{-}\right)\right)-\varphi\left(\frac{1}{2}\left(u_{-}-u_{+}\right)\right)=0 .
$$

This is why formula (1.2) defines a weak solution $U$ of the Cauchy problem (1.1). Moreover, the function $U$ is an entropy solution, since it verifies the Lax entropy condition [13]:

$$
f^{\prime}\left(u_{-}\right) \geqslant \lambda \geqslant f^{\prime}\left(u_{+}\right) .
$$

Indeed, by (1.3) and (1.4),

$$
\begin{aligned}
f^{\prime}\left(u_{+}\right) & =\lambda+\varphi^{\prime}\left(u_{+}-\beta\right), \\
\varphi^{\prime}\left(u_{+}-\beta\right) & \equiv \varphi^{\prime}\left(\frac{1}{2}\left(u_{+}-u_{-}\right)\right) \\
& \leqslant \varphi^{\prime}(0)=0 \leqslant \varphi^{\prime}\left(u_{-}-\beta\right) \equiv \varphi^{\prime}\left(\frac{1}{2}\left(u_{-}-u_{+}\right)\right) .
\end{aligned}
$$

So inequalities (1.6) follow.

Observe that the flux function $f=\frac{1}{2} u^{2}$ in the Hopf equation

$$
u_{t}+u u_{x}=0
$$

satisfies conditions (1.4) with $\lambda=\beta$ and $\varphi(u)=\frac{1}{2} u^{2}-\frac{1}{2} \beta^{2}$.

Let us consider the parabolic problem

$$
u_{t}+f(u)_{x}=\varepsilon u_{x x},\left.\quad u\right|_{t=0}=u_{0}^{\varepsilon}(x),
$$


where $u_{0}^{\varepsilon}(x)$ is a smooth function such that

$$
u_{0}^{\varepsilon}-u_{0} \rightarrow 0 \quad \text { in } L_{\mathrm{loc}}^{1}(\mathbb{R}) \text { as } \varepsilon \downarrow 0
$$

and

$$
\left\|u_{0}^{\varepsilon}\right\|_{\infty} \leqslant c, \quad\left\|\frac{\mathrm{d} u_{0}^{\varepsilon}}{\mathrm{d} x}, \varepsilon \frac{\mathrm{d}^{2} u_{0}^{\varepsilon}}{\mathrm{d} x^{2}}\right\|_{1} \leqslant c
$$

uniformly in $\varepsilon$. Here and in what follows, $c$ denotes different constants which do not depend on $\varepsilon$, and $\|\cdot\|_{p}$ stands for the norm in $L^{p}(\mathbb{R})$.

Naturally, one expects that problem (1.8) approximates problem (1.1) for small $\varepsilon$. This means that the difference $|u-U|$ is small far from $\Gamma$,

$$
u \simeq u_{-} \text {to the left of } \Gamma, \quad u \simeq u_{+} \text {to the right of } \Gamma,
$$

and there is a shock layer centred at $\Gamma$, where $u(t, x)$ changes smoothly in value from $u_{-}$ to $u_{+}$when $t$ is fixed and $x$ crosses the curve $\Gamma$ from the left.

The goal of this paper is to formalize the notion of a shock layer when the viscous problem (1.8) approximates the hyperbolic problem (1.1). In doing so, we are motivated by the Mises conjecture and the observation that the viscosity effect reveals itself strongly only within the shock layer.

Consider the cylinder

$$
\Omega_{\delta}=\{(t, x):|x-\lambda t| \leqslant \delta, 0<t<T\}
$$

centred at the shock curve $\Gamma$. We are looking for the thickness $\delta$ as a function of $\varepsilon$ to meet the following definition.

Definition 1.1. We call a function $\delta(\varepsilon), 0<\varepsilon<1$, a shock-layer thickness (SL thickness) if there is a sequence of initial data $u_{0}^{\varepsilon}(x), \varepsilon \downarrow 0$, which meets conditions (1.9) and (1.10) and is such that

(1) $0 \leqslant \delta(\varepsilon) \downarrow 0$ as $\varepsilon \downarrow 0$;

(2) for any compact $K \subset \mathbb{R}$

$$
\lim _{\varepsilon \rightarrow 0} \sup _{0<t<T}\|u-U\|_{L^{\infty}\left(K \backslash \Omega_{\delta(\varepsilon)}^{t}\right)}=0
$$

where

$$
\Omega_{\delta}^{s}=\Omega_{\delta} \cap\{t=s\}
$$

(3) the limit inequality

$$
\liminf _{\varepsilon \rightarrow 0} \sup _{0<t<T}\|u-U\|_{L^{\infty}(\mathbb{R})}>0
$$

is valid. 
Clearly, $\delta(\varepsilon)$ is not defined uniquely. Indeed, given an SL thickness $\delta(\varepsilon)$, any function $\delta_{1}(\varepsilon)$ is also an SL thickness if $\delta(\varepsilon) \leqslant \delta_{1}(\varepsilon)$ and $\delta_{1}(\varepsilon) \downarrow 0$ as $\varepsilon \downarrow 0$. Thus, the true SL thickness $\delta_{*}(\varepsilon)$ should be defined as

$$
\delta_{*}(\varepsilon)=\inf _{\alpha} \delta_{\alpha}(\varepsilon)
$$

where $\delta_{\alpha}(\varepsilon), \alpha \in I$, is a family of SL thicknesses.

Our main result is the following theorem.

Theorem 1.2. Let conditions (1.3), (1.4) be satisfied. Then any function $\delta(\varepsilon)$, $0<\varepsilon<1$, is an SL thickness if $\delta \asymp \sqrt{\varepsilon}$. The last relationship has the meaning

$$
0 \leqslant \delta(\varepsilon) \downarrow 0 \quad \text { and } \quad \frac{\sqrt{\varepsilon}}{\delta(\varepsilon)} \rightarrow 0 \quad \text { as } \varepsilon \downarrow 0 .
$$

The formulated result can be treated as an attempt to define a shock layer as a domain which depends on the vanishing viscosity optimally in the sense that it is the thinnest domain where solutions of the parabolic problem fail to approximate the shock solution of the limit problem in the $L^{\infty}$-norm. The same approach has been applied to define a boundary layer for scalar conservation laws [2] and a shear-viscosity boundary layer in a boundary-value problem for the Navier-Stokes equations of compressible fluids [1].

Example (4.1) given in $\S 4$ proves that the law $\delta \asymp \sqrt{\varepsilon}$ is optimal, as long as the Riemann problem (1.1) meets conditions (1.3) and (1.4). Nevertheless, the law $\delta \asymp \varepsilon$ may occur as in example (4.5). In particular, the law $\delta \asymp \varepsilon$ can be derived if one applies the above definition of the SL thickness $\delta$ to the results on asymptotic behaviour of 'viscous solutions' near the shock obtained by the method of matched asymptotic expansions [3, $4,8]$ under some hypotheses, with the condition $f^{\prime \prime}(u)>0$ being principal. (Observe that the claim of Theorem 1.2 is conditioned by the non-strict inequality $f^{\prime \prime}(u) \geqslant 0$.)

There is no evidence to support the law $\delta \asymp \varepsilon^{\alpha}$ with $\alpha$ different from $\frac{1}{2}$ and 1 . It is remarked in [14] that the exponents $\alpha=\frac{1}{2}$ and $\alpha=1$ correspond to the characteristic and non-characteristic shocks, respectively. However, the example [4] of the transition boundary layer $\varepsilon \lesssim x \lesssim \varepsilon^{2 / 3}$ in the Cauchy problem for the ordinary differential equation

$$
\varepsilon \frac{\mathrm{d} u}{\mathrm{~d} x}=f(x, u)
$$

indicates that a possibility exists for a shock layer of thickness $\delta \asymp \varepsilon^{\alpha}$ with $\alpha \neq \frac{1}{2}$ and $\alpha \neq 1$ as far as a non-homogeneous conservation law

$$
U_{t}+f(x, U)_{x}=0
$$

is concerned.

In the theory of hyperbolic equations, shock-layer-thickness estimates find applications in constructing finite-difference schemes which take into account the artificial viscosity effect $[\mathbf{1 1}]$. 


\section{Parabolic problems}

Here we discuss some properties of the parabolic problem (1.8). First, we construct initial data $u_{0}^{\varepsilon}(x)$ which approximate $u_{0}(x)$ in the sense of (1.9) and (1.10). Define

$$
U_{0}^{\varepsilon}(x)=\beta+\left\{\left(u_{0}(x)-\beta\right) \mathbf{1}_{|x| \leqslant 1 / \varepsilon}\right\} * \rho_{\varepsilon}(x) .
$$

Here, $\mathbf{1}_{\Omega}(x)$ is the characteristic function of a set $\Omega \subset \mathbb{R}$ and $\rho_{\varepsilon}$ is a symmetric mollifier, i.e. $\rho(-x)=\rho(x), 0 \leqslant \rho \in \mathcal{D}(\mathbb{R})$, and

$$
\rho_{\varepsilon}(x)=\frac{1}{\varepsilon} \rho\left(\frac{|x|}{\varepsilon}\right), \quad \int_{\mathbb{R}} \rho \mathrm{d} x=1, \quad \operatorname{supp} \rho \subseteq\{x:|x| \leqslant 1\} .
$$

Lemma 2.1. The function $w_{0}^{\varepsilon}(x) \equiv U_{0}^{\varepsilon}(x)-\beta$ has the properties that it is odd and that

$$
\left\|\frac{\mathrm{d} w_{0}^{\varepsilon}}{\mathrm{d} x}, \varepsilon \frac{\mathrm{d}^{2} w_{0}^{\varepsilon}}{\mathrm{d} x^{2}}\right\|_{1} \leqslant c\left(u_{-}-u_{+}\right) .
$$

The function $U_{0}^{\varepsilon}(x)$ defined by (2.1) satisfies properties (1.9) and (1.10).

Proof. The function $v_{0}^{\varepsilon}(x)=\left(u_{0}-\beta\right) \mathbf{1}_{|x| \leqslant 1 / \varepsilon}$ is odd and the mollifier $\rho_{\varepsilon}(x)$ is symmetric, hence the convolution $v_{0}^{\varepsilon} * \rho_{\varepsilon}$ is also odd.

To prove estimates $(2.2)$, we calculate the $L^{1}$-norm through the total variation. We have

$$
\left\|\frac{\mathrm{d} w_{0}^{\varepsilon}}{\mathrm{d} x}\right\|_{1}=\operatorname{TV}\left(w_{0}^{\varepsilon}\right)=\sup _{\varphi} \int_{\mathbb{R}} w_{0}^{\varepsilon} \varphi_{x} \mathrm{~d} x
$$

where 'sup' is taken with respect to $\varphi(x)$ such that $\varphi \in \mathcal{D}(\mathbb{R})$ and $\|\varphi\|_{\infty} \leqslant 1$. On the other hand,

$$
\int_{\mathbb{R}} w_{0}^{\varepsilon} \varphi_{x} \mathrm{~d} x=\int_{\mathbb{R}} v_{0}^{\varepsilon}(x) \frac{\mathrm{d}}{\mathrm{d} x}\left(\varphi * \rho_{\varepsilon}\right) \mathrm{d} x \leqslant \mathrm{TV}\left(v_{0}^{\varepsilon}\right)=2\left(u_{-}-u_{+}\right) .
$$

Hence, the first estimate in (2.2) is proved.

Next we have

$$
\begin{aligned}
\left\|\frac{\mathrm{d}^{2} w_{0}^{\varepsilon}}{\mathrm{d} x^{2}}\right\|_{1} & =\sup _{\psi} \frac{\int_{\mathbb{R}} \psi\left(\mathrm{d}^{2} / \mathrm{d} x^{2}\right) w_{0}^{\varepsilon} \mathrm{d} x}{\|\psi\|_{\infty}}=\sup _{\psi} \frac{\int_{\mathbb{R}} v_{0}^{\varepsilon}\left(\rho_{\varepsilon} * \psi\right)^{\prime \prime} \mathrm{d} x}{\|\psi\|_{\infty}} \\
& \leqslant \operatorname{TV}\left(v_{0}^{\varepsilon}\right) \sup _{\psi} \frac{\left\|\left(\rho_{\varepsilon} * \psi\right)^{\prime \prime}\right\|_{\infty}}{\|\psi\|_{\infty}} \leqslant \frac{c}{\varepsilon} \operatorname{TV}\left(v_{0}^{\varepsilon}\right),
\end{aligned}
$$

where 'sup' is taken with respect to $\psi \in L^{\infty}(\mathbb{R})$ such that $\psi \neq 0$. This implies the second estimate in (2.2). All the other claims of the lemma are trivial.

The function $w=u-\beta$ solves the Cauchy problem

$$
w_{t}+f(w+\beta)_{x}=\varepsilon w_{x x},\left.\quad w\right|_{t=0}=w_{0}^{\varepsilon}(x),
$$

with $f(u) \in C^{4}(\mathbb{R})$ and with the initial data

$$
w_{0}^{\varepsilon} \in L^{\infty}(\mathbb{R}) \cap L^{1}(\mathbb{R}) \cap \mathcal{D}(\mathbb{R}) .
$$


Hence, the theory of parabolic differential equations is applied $[\mathbf{7}, \mathbf{9}]$ to conclude that there is a unique solution $w$ of (2.3) such that

$$
\begin{gathered}
w \in L^{2}\left(0, T ; W^{5,2}(\mathbb{R})\right) \cap C\left(0, T ; W^{4,2}(\mathbb{R})\right), \\
\frac{\partial^{k} w}{\partial t^{k}} \in L^{2}\left(0, T ; W^{5-2 k, 2}(\mathbb{R})\right) \cap C\left(0, T ; W^{4-2 k, 2}(\mathbb{R})\right), \quad 2 k \leqslant 4 .
\end{gathered}
$$

Moreover, the following estimates are valid uniformly in $\varepsilon$ :

$$
\begin{gathered}
\|w(t)\|_{\infty} \leqslant\left\|w_{0}^{\varepsilon}\right\|_{\infty}, \quad\left\|w_{x}(t)\right\|_{1} \leqslant\left\|\frac{\mathrm{d}}{\mathrm{d} x} w_{0}^{\varepsilon}\right\|_{1}, \\
\left\|\frac{\partial}{\partial t} w(t)\right\|_{1} \leqslant M\left\|\frac{\mathrm{d}}{\mathrm{d} x} w_{0}^{\varepsilon}\right\|_{1}+\varepsilon\left\|\frac{\mathrm{d}^{2}}{\mathrm{~d} x^{2}} w_{0}^{\varepsilon}\right\|_{1}
\end{gathered}
$$

where

$$
M=\sup \left\{\left|f^{\prime}(u)\right|,|u| \leqslant \max \left\{\left|u_{-}\right|,\left|u_{+}\right|\right\}\right\} .
$$

The first estimate in (2.4) is due to the maximum principle. The other estimates can be derived by the Kružkov technique [5]. We recall briefly the basic idea, since it underlies the proof of Theorem 1.2.

Let us differentiate equation (2.3) with respect to $x$ and multiply the result by

$$
\psi_{R}(x) \operatorname{sgn}\left(\frac{\partial w}{\partial x}\right), \quad R>1
$$

where $\psi_{R}(x)=\psi(|x| / R)$ and

$$
\psi(s)= \begin{cases}1, & s \in\left[0, \frac{1}{2}\right], \\ \text { non-negative polynomial, } & s \in\left[\frac{1}{2}, 1\right], \\ \exp (-s), & s \geqslant 1 .\end{cases}
$$

Calculations give

$$
\begin{aligned}
\frac{\mathrm{d}}{\mathrm{d} t} \int_{\mathbb{R}} \psi_{R}\left|\frac{\partial w}{\partial x}\right| \mathrm{d} x & =\int_{\mathbb{R}} \frac{\partial \psi_{R}}{\partial x}\left|\frac{\partial w}{\partial x}\right| f^{\prime}(u)+\varepsilon \psi_{R} \frac{\partial^{3} w}{\partial x^{3}} \operatorname{sgn}\left(\frac{\partial w}{\partial x}\right) \mathrm{d} x \\
& \leqslant \int_{\mathbb{R}} \frac{\partial \psi_{R}}{\partial x}\left|\frac{\partial w}{\partial x}\right| f^{\prime}(u)+\varepsilon\left|\frac{\partial w}{\partial x}\right| \frac{\partial^{2} \psi_{R}}{\partial x^{2}} \mathrm{~d} x
\end{aligned}
$$

From the inequalities

$$
\left|\frac{\partial \psi_{R}}{\partial x}(x)\right| \leqslant \frac{c}{R} \psi_{R}(x), \quad\left|\frac{\partial^{2} \psi_{R}}{\partial x^{2}}(x)\right| \leqslant \frac{c}{R^{2}} \psi_{R}(x),
$$

we obtain

$$
\frac{\mathrm{d}}{\mathrm{d} t} \int_{\mathbb{R}} \psi_{R}\left|\frac{\partial w}{\partial x}\right| \mathrm{d} x \leqslant \frac{c}{R} \int_{\mathbb{R}} \psi_{R}\left|\frac{\partial w}{\partial x}\right| \mathrm{d} x
$$


Now, the second estimate in (2.4) follows if one integrates (2.7) and sends $R$ to $\infty$. In order to prove (2.5), one should differentiate (2.3) with respect to $t$ and multiply by

$$
\psi_{R}(x) \operatorname{sgn}\left(\frac{\partial w}{\partial t}\right)
$$

In a similar way one obtains

$$
\left\|\frac{\partial w}{\partial t}(t)\right\|_{1} \leqslant\left\|\frac{\partial w}{\partial t}(0)\right\|_{1}
$$

The solution $w$ is regular enough to make sure that $(\partial w / \partial t)(0)$ may be written

$$
\frac{\partial w}{\partial t}(0)=\varepsilon \frac{\mathrm{d}^{2} w_{0}}{\mathrm{~d} x^{2}}-f^{\prime}\left(u_{0}\right) \frac{\mathrm{d} w_{0}}{\mathrm{~d} x} .
$$

Thus, the estimate (2.5) follows.

Let $K$ be a closed bounded interval in $\mathbb{R}$. From estimates (2.4) and (2.5) and the compact imbedding of $W^{1,1}(K)$ into $L^{1}(K)$, one can apply the Arzel-Ascoli Theorem to conclude that the sequence $w^{\varepsilon}(t, x) \equiv w(t, x)$ is compact in $C\left([0, T] ; L_{\mathrm{loc}}^{1}(K)\right)$. Since $U$ is the unique entropy solution of problem (1.1), we arrive at the following conclusion.

Theorem 2.2. Let conditions (1.3) and (1.4) be satisfied. Then

$$
\lim _{\varepsilon \rightarrow 0}\|u-U\|_{C\left(0, T ; L_{1 \mathrm{loc}}^{1}(\mathbb{R})\right)}=0
$$

and the estimates

$$
\sup _{0<t<T}\|u(t)\|_{\infty} \leqslant c, \quad \sup _{0<t<T}\left\|\frac{\partial u}{\partial x}(t)\right\|_{1} \leqslant c
$$

hold uniformly in $\varepsilon$.

\section{Shock-layer thickness}

Here we prove Theorem 1.2 assuming that the initial data $u_{0}^{\varepsilon}(x)$ are given by (2.1). Let us introduce a cut-off function $\xi_{\delta}(t, x)$ for the cylinder $\Omega_{\delta}$. First, we define an even function $\chi_{\delta}(x)$ for non-negative values of $x$ by the formula

$$
\chi_{\delta}(x)= \begin{cases}\frac{2}{\delta^{2}} x^{2}, & 0 \leqslant x \leqslant \frac{1}{2} \delta, \\ 1-\frac{2}{\delta^{2}}(x-\delta)^{2}, & \frac{1}{2} \delta \leqslant x \leqslant \delta, \\ 1, & x \geqslant \delta .\end{cases}
$$

Next, we define $\xi_{\delta}(t, x)=\chi_{\delta}(x-\lambda t)$. Clearly,

$$
\left.\begin{array}{c}
0 \leqslant \xi_{\delta}(t, x) \leqslant 1, \quad \xi_{\delta}(t, \lambda t)=0, \quad \xi_{\delta}(t, x)=1 \quad \text { for }|x-\lambda t| \geqslant \delta, \\
\xi_{\delta} \in C^{1}(\mathbb{R} \times \mathbb{R}) \cap W_{\text {loc }}^{2,2}(\mathbb{R} \times \mathbb{R}), \quad\left|\frac{\partial \xi_{\delta}}{\partial x}\right| \leqslant \frac{2}{\delta}, \quad\left|\frac{\partial^{2} \xi_{\delta}}{\partial x^{2}}\right| \leqslant \frac{4}{\delta^{2}} .
\end{array}\right\}
$$

The next assertion is a crucial one in the proof of Theorem 1.2. 
Lemma 3.1. There is a constant $\kappa$ independent of $\varepsilon$ such that

$$
\begin{array}{ll}
u-\beta \leqslant \kappa(x-\lambda t) & \text { for } x-\lambda t \geqslant 0, \\
u-\beta \geqslant \kappa(x-\lambda t) & \text { for } x-\lambda t \leqslant 0 .
\end{array}
$$

Proof. By the change of variables

$$
w=u-\beta, \quad \alpha=x-\lambda t
$$

we reduce problem (1.8) to

$$
w_{t}+\varphi(w)_{\alpha}=\varepsilon w_{\alpha \alpha},\left.\quad w\right|_{t=0}=w_{0}^{\varepsilon}(\alpha) \equiv U_{0}^{\varepsilon}(\alpha)-\beta .
$$

Because $w_{0}^{\varepsilon}(\alpha)$ is odd and $\varphi(w)$ is even, one can prove, using uniqueness of the solution to problem $(3.2)$ that the function $w(t, \alpha)$ is odd with respect to $\alpha$. So, it is enough to prove the first inequality of the lemma.

Given a positive constant $\kappa$, the function $v=\kappa \alpha-w$ solves the problem

$$
v_{t}+\varphi^{\prime}(w) v_{\alpha}-\kappa \varphi^{\prime}(w)=\varepsilon v_{\alpha \alpha},\left.\quad v\right|_{t=0}=\kappa \alpha-w_{0}^{\varepsilon}(\alpha),\left.\quad v\right|_{\alpha=0}=0
$$

in the domain

$$
Q=\{(t, \alpha) ; 0<t<T, \alpha>0\}
$$

Clearly, $v(0, \alpha) \geqslant 0$ for some $\kappa>0$. Suppose $v$ achieves a negative minimum in the interior of $Q$, then the function $z=v \exp (-p t)$ also has this property and

$$
z_{t}+p z+\varphi^{\prime}(w) z_{\alpha}-\kappa \varphi^{\prime}(w) \mathrm{e}^{-p t}=\varepsilon z_{\alpha \alpha},\left.\quad z\right|_{t=0, \alpha \geqslant 0} \geqslant 0,\left.\quad z\right|_{\alpha=0}=0 .
$$

This is a contradiction, since $\varphi^{\prime \prime} \geqslant 0$ and

$$
\varphi^{\prime}(w)=\varphi^{\prime \prime}(\bar{w}) w=\varphi^{\prime \prime}(\bar{w})\left(\kappa \alpha-z \mathrm{e}^{p t}\right),
$$

where $\bar{w}$ lies between 0 and $w$.

The next assertion is a consequence.

Lemma 3.2. There is a constant $\gamma$ independent of $\varepsilon$ such that

$$
\left(f^{\prime}(u)-\lambda\right) \frac{\partial \xi_{\delta}}{\partial x} \leqslant \gamma \xi_{\delta} \quad \text { for }|x-\lambda t| \leqslant \delta
$$

Proof. Denote

$$
M_{1}=1+\sup _{|u| \leqslant 1} \varphi^{\prime \prime}(\kappa u)
$$

If $x-\lambda t>0$, we have, by Lemma 3.1,

$$
f^{\prime}(u)-\lambda=\varphi^{\prime}(u-\beta) \leqslant \varphi^{\prime}(\kappa(x-\lambda t))=\varphi^{\prime \prime}(\bar{u}) \kappa(x-\lambda t) \leqslant M_{1}(x-\lambda t) .
$$

Similarly, if $x-\lambda t<0$, we have

$$
f^{\prime}(u)-\lambda=\varphi^{\prime}(u-\beta) \geqslant \varphi^{\prime}(\kappa(x-\lambda t)) \geqslant M_{1}(x-\lambda t) .
$$


Now, one should calculate the product

$$
\alpha \chi_{\delta}(\alpha) \equiv(x-\lambda t) \xi_{\delta}(x-\lambda t), \quad \alpha=x-\lambda t,
$$

on the intervals

$$
-\delta<\alpha<-\frac{1}{2} \delta, \quad-\frac{1}{2} \delta<\alpha<0, \quad 0<\alpha<\frac{1}{2} \delta, \quad \frac{1}{2} \delta<\alpha<\delta
$$

to verify that inequality (3.3) holds with $\gamma=2 M_{1}$.

The principal step in the proof of Theorem 1.2 is to obtain estimates of the integral

$$
p(t ; \varepsilon, \delta, K) \equiv \int_{K \backslash \Omega_{\delta}^{t}}\left|u_{x}(t)\right| \mathrm{d} x,
$$

via $\varepsilon$, where $K$ is compact in $\mathbb{R}$. To this end, we modify the Kružkov technique.

Lemma 3.3. There is a constant $c$ such that

$$
J_{R}(t) \equiv \int_{\mathbb{R}}\left|u_{x}(t)\right| \psi_{R} \xi_{\delta} \mathrm{d} x \leqslant \mathrm{e}^{(\gamma+(c / R)) T}\left[J_{R}(0)+c \varepsilon\left(\frac{1}{R^{2}}+\frac{1}{R \delta}+\frac{1}{\delta^{2}}\right)\right] .
$$

Proof. If $\eta$ is a small real number, the function $\varphi_{\eta}(z)=\left(z^{2}+\eta^{2}\right)^{1 / 2}$ is a smooth approximation of $|z|$ and

$$
\varphi_{\eta}^{\prime \prime}(z) z^{2} \leqslant \eta, \quad z \varphi_{\eta}^{\prime}(z) \rightarrow|z| \quad \text { as } \eta \rightarrow 0 .
$$

Denoting $u_{x}=z$, we differentiate equation (1.8) with respect to $x$, multiply by $\varphi_{\eta}^{\prime}(z) \psi_{R}(x) \xi_{\delta}(t, x)$ and integrate with respect to $x$ and $t$. Let us consider the resulting terms:

$$
\begin{aligned}
& \int_{0}^{t} \int_{\mathbb{R}} z_{t} \varphi_{\eta}^{\prime} \psi_{R} \xi_{\delta} \mathrm{d} x \mathrm{~d} s=\left.\int_{\mathbb{R}} \varphi_{\eta} \psi_{R} \xi_{\delta} \mathrm{d} x\right|_{0} ^{t}+\lambda \int_{0}^{t} \int_{\mathbb{R}} \varphi_{\eta} \psi_{R} \frac{\partial \xi_{\delta}}{\partial x} \mathrm{~d} x \mathrm{~d} s \\
& \int_{0}^{t} \int_{\mathbb{R}}\left(f^{\prime}(u) z-\varepsilon z_{x}\right)_{x} \varphi_{\eta}^{\prime} \psi_{R} \xi_{\delta} \mathrm{d} x \mathrm{~d} s \\
&=\int_{0}^{t} \int_{\mathbb{R}} \varphi_{\eta}^{\prime \prime} \psi_{R} \xi_{\delta}\left[\sqrt{\varepsilon} z_{x}-\frac{z f^{\prime}(u)}{2 \sqrt{\varepsilon}}\right]^{2} \mathrm{~d} x \mathrm{~d} s \\
& \quad-\frac{1}{4 \varepsilon} \int_{0}^{t} \int_{\mathbb{R}} \varphi_{\eta}^{\prime \prime} \psi_{R} \xi_{\delta} z^{2}\left|f^{\prime}(u)\right|^{2}+4 \varepsilon f^{\prime}(u) z \varphi_{\eta}^{\prime}(z) \xi_{\delta} \frac{\partial \psi_{R}}{\partial x} \mathrm{~d} x \mathrm{~d} s \\
& \quad-\int_{0}^{t} \int_{\mathbb{R}} f^{\prime}(u) z \varphi_{\eta}^{\prime}(z) \psi_{R} \frac{\partial \xi_{\delta}}{\partial x}+\varepsilon \varphi_{\eta}(z) \frac{\partial^{2} \xi_{\delta} \psi_{R}}{\partial x^{2}} \mathrm{~d} x \mathrm{~d} s .
\end{aligned}
$$

In the limit as $\eta \rightarrow 0$, we obtain

$$
\begin{aligned}
J_{R}(t) \leqslant J_{R}(0)+\int_{0}^{t} \int_{\mathbb{R}}\left(f^{\prime}(u)-\lambda\right)|z| \psi_{R} \frac{\partial \xi_{\delta}}{\partial x} & \\
& +f^{\prime}(u)|z| \frac{\partial \psi_{R}}{\partial x} \xi_{\delta} \mathrm{d} x \mathrm{~d} s+\varepsilon \int_{0}^{t} \int_{\mathbb{R}}|z| \frac{\partial^{2} \psi_{R} \xi_{\delta}}{\partial x^{2}} \mathrm{~d} x \mathrm{~d} s .
\end{aligned}
$$


We rewrite this inequality taking into account the estimates of Theorem 2.2 , inequalities (2.5), (3.1) and (3.3):

$$
J_{R}(t) \leqslant J_{R}(0)+\left(\gamma+\frac{c}{R}\right) \int_{0}^{t} J_{R}(s) \mathrm{d} s+\varepsilon c\left(\frac{1}{R^{2}}+\frac{1}{R \delta}+\frac{1}{\delta^{2}}\right) .
$$

Applying the Gronwall Lemma, we arrive at (3.4).

Lemma 3.4. Given compact $K \subset \mathbb{R}$, we have

$$
\sup _{0<t<T} p(t ; \varepsilon, \delta(\varepsilon), K) \rightarrow 0 \quad \text { as } \varepsilon \downarrow 0,
$$

for any function $\delta(\varepsilon)$ such that $\varepsilon / \delta^{2}(\varepsilon) \rightarrow 0$.

Proof. Let us choose $R$ such that $K \subseteq\left[-\frac{1}{2} R, \frac{1}{2} R\right]$ and let $\delta(\varepsilon)$ be a function which satisfies the conditions of the lemma. Since

$$
\operatorname{supp} \frac{\partial U_{0}^{\varepsilon}}{\partial x} \subseteq\{|x| \leqslant \varepsilon\} \cup\left\{\left|x-\frac{1}{\varepsilon}\right| \leqslant \varepsilon\right\} \cup\left\{\left|x+\frac{1}{\varepsilon}\right| \leqslant \varepsilon\right\},
$$

there is a small $\varepsilon_{1}$ depending on $R$ such that

$$
\begin{aligned}
J_{R}(0)= & \int_{-\varepsilon}^{\varepsilon}\left|\frac{\partial U_{0}^{\varepsilon}}{\partial x}\right| \chi_{\delta(\varepsilon)}(x) \mathrm{d} x+\int_{|x-(1 / \varepsilon)| \leqslant \varepsilon}\left|\frac{\partial U_{0}^{\varepsilon}}{\partial x}\right| \mathrm{e}^{-|x| / R} \mathrm{~d} x \\
& +\int_{|x+(1 / \varepsilon)| \leqslant \varepsilon}\left|\frac{\partial U_{0}^{\varepsilon}}{\partial x}\right| \mathrm{e}^{-|x| / R} \mathrm{~d} x \\
& \leqslant c \sup _{|x| \leqslant \varepsilon}\left|\chi_{\delta(\varepsilon)}(x)\right|+c \mathrm{e}^{-(1 / R)((1 / \varepsilon)-\varepsilon)}
\end{aligned}
$$

for any $\varepsilon \leqslant \varepsilon_{1}$. Clearly, there is a small $\varepsilon_{2}, \varepsilon_{2} \leqslant \varepsilon_{1}$, such that

$$
\varepsilon \leqslant \frac{1}{2} \delta^{2}(\varepsilon) \leqslant \frac{1}{2} \delta(\varepsilon) \quad \text { for } \varepsilon \leqslant \varepsilon_{2} .
$$

Hence, by the definition of $\chi_{\delta}(x)$,

$$
\sup _{|x| \leqslant \varepsilon}\left|\chi_{\delta(\varepsilon)}(x)\right| \leqslant \chi_{\delta(\varepsilon)}(\varepsilon) \leqslant \frac{2}{\delta^{2}(\varepsilon)} \varepsilon^{2} \leqslant \varepsilon \quad \text { for } \varepsilon \leqslant \varepsilon_{2} .
$$

Now, it follows from estimate (3.4) that

$$
p(t ; \varepsilon, \delta(\varepsilon), K) \leqslant c \varepsilon\left(1+\frac{1}{\delta^{2}(\varepsilon)}\right)+c \mathrm{e}^{-(1 / R)((1 / \varepsilon)-\varepsilon)}
$$

for $\varepsilon \leqslant \varepsilon_{2}$. This proves the lemma.

Proof of Theorem 1.2. Let $K$ be compact such that $\Omega_{1}^{t} \subseteq K$ for all $t \in[0, T]$. Let $x, y \in K$ and $x>y>\lambda t+\delta$. Then

$$
u(t, x)-U(t, x)=u(t, y)-U(t, y)+\int_{y}^{x} u_{x} \mathrm{~d} \zeta .
$$


By integrating with respect to $y$ from $\lambda t+\delta$ to $\lambda t+\delta+1$, we obtain

$$
|u(t, x)-U(t, x)| \leqslant\|u(t)-U(t)\|_{L_{\mathrm{loc}}^{1}(\mathbb{R})}+\int_{K \backslash \Omega_{\delta}^{t}}\left|u_{x}(t)\right| \mathrm{d} x .
$$

Similarly, this inequality can be proved for $x<\lambda t-\delta$. Hence, by Theorem 2.2 and Lemma 3.4,

$$
\sup _{0<t<T}\|u-U\|_{L^{\infty}\left(K \backslash \Omega_{\delta(\varepsilon)}^{t}\right)} \rightarrow 0 \quad \text { as } \varepsilon \rightarrow 0 .
$$

The third condition in the definition of a shock layer holds due to the equality $u(t, \lambda t)=\beta$ for each $\varepsilon>0$. Thus, Theorem 1.2 is proved.

\section{Examples}

One might wonder if the shock-layer estimate $\delta \asymp \sqrt{\varepsilon}$ given by Theorem 1.2 is optimal. Our first example below serves to prove that this result cannot be improved in the class of fluxes $f(u)$ given by (1.3) and (1.4). The second example below is concerned with the case when the estimate can be improved up to the law $\delta \asymp \varepsilon$. In searching for a reason why these two laws of thickness of the shock layer occur, we show that the shock is characteristic in the first example and non-characteristic in the second example. We recall that a shock is called characteristic if some characteristic curves touch it, and a shock is non-characteristic if all the characteristic curves cross it [13].

Example 4.1. First we consider the Riemann problem

$$
U_{t}=0,\left.\quad U\right|_{t=0}=u_{0}(x)
$$

Here, the shock is characteristic and coincides with the line $x=0$.

One can write two approximations for (4.1):

$$
u_{1 t}=\varepsilon u_{1 x x},\left.\quad u_{1}\right|_{t=0}=U_{0}^{\varepsilon}(x) \equiv \beta+\left\{\left(u_{0}(x)-\beta\right) \mathbf{1}_{|x| \leqslant 1 / \varepsilon}\right\} * \rho_{\varepsilon}(x)
$$

and

$$
u_{2 t}=\varepsilon u_{2 x x},\left.\quad u_{2}\right|_{t=0}=u_{0}(x) .
$$

Clearly, $u_{2}$ is a self-similar solution, and it is given by the representation formula

$$
u_{2}=u_{-}+\frac{u_{+}-u_{-}}{F(\infty)} F(\theta), \quad F(\theta)=\int_{-\infty}^{\theta} \mathrm{e}^{-s^{2}} \mathrm{~d} s, \quad \theta=\frac{x}{\sqrt{4 \varepsilon t}} .
$$

It follows that for any fixed $t>0$,

$$
\liminf _{\varepsilon \rightarrow 0} \sup _{|x| \leqslant \delta}\left|U-u_{2}\right|>0 \quad \text { if } \delta=M \sqrt{\varepsilon}
$$

Since $u_{2}$ does not meet the regularity conditions of Theorem 1.2 , one cannot use this approximation directly to conclude that the law $\delta=\delta(\varepsilon)$ given by Theorem 1.2 is optimal. 
We justify this conclusion by the comparison of $u_{2}$ with $u_{1}$. Denoting $z=u_{1}-u_{2}$, we write $z$ as the Poisson integral

$$
z=\frac{1}{\sqrt{4 \pi \varepsilon t}}\{\int_{|y| \leqslant \varepsilon} \underbrace{\mathrm{e}^{-\left((x-y)^{2} / 4 \varepsilon t\right)} z_{0}(y)} \mathrm{d} y+\int_{|y| \geqslant(1 / \varepsilon)-\varepsilon} \underbrace{\cdots} \mathrm{d} y\} \equiv z_{1}+z_{2},
$$

using the following property of $\left.z_{0} \equiv\left(u_{1}-u_{2}\right)\right|_{t=0}$ :

$$
\operatorname{supp} z_{0} \cap\left\{\varepsilon \leqslant|x| \leqslant \frac{1}{\varepsilon}-\varepsilon\right\}=\emptyset \text {. }
$$

Note that

$$
\left|z_{1}\right| \leqslant \frac{c \varepsilon}{\sqrt{\varepsilon t}} \mathrm{e}^{-\left((\eta \varepsilon-x)^{2} / 4 \varepsilon t\right)}, \quad|\eta| \leqslant 1 .
$$

Hence, $\left|z_{1}\right| \leqslant c \sqrt{\varepsilon}$ uniformly in

$$
Q_{\tau, R}=\{(t, x) \in[\tau, T] \times[-R, R]\}, \quad \tau>0 .
$$

The second term $z_{2}$ is bounded by

$$
\left|z_{2}\right| \leqslant c\left\{\int_{-\infty}^{-\sigma(\varepsilon)} \mathrm{e}^{-s^{2}} \mathrm{~d} s+\int_{\sigma(\varepsilon)}^{+\infty} \mathrm{e}^{-s^{2}} \mathrm{~d} s\right\}
$$

uniformly in $Q_{\tau, R}$, where $\sigma(\varepsilon) \rightarrow \infty$ as $\varepsilon \rightarrow 0$. Thus,

$$
\left|u_{1}-u_{2}\right| \rightarrow 0 \text { uniformly in } Q_{\tau, R} \text { as } \varepsilon \rightarrow 0 .
$$

The last convergence result combined with (4.4) yields the limit inequality

$$
\liminf _{\varepsilon \rightarrow 0} \sup _{\tau \leqslant t \leqslant T}\left\|U-u_{1}\right\|_{L^{\infty}\left(K \backslash \Omega_{\delta}\right)}>0, \quad \delta=M \sqrt{\varepsilon},
$$

for any $M>0$ and any compact $K=[-R, R]$. This proves that Theorem 1.2 is optimal.

Example 4.2. Consider the Riemann problem

$$
U_{t}+f(U)_{x}=0,\left.\quad U\right|_{t=0}=u_{0}(x),
$$

with the flux $f(u)$, the data $u_{ \pm}$, and the shock $x=\lambda t$ such that the boundary-value problem

$$
-\lambda \frac{\mathrm{d} w}{\mathrm{~d} x}+\frac{\mathrm{d} f(w)}{\mathrm{d} x}=\varepsilon_{0} \frac{\mathrm{d}^{2} w}{\mathrm{~d} x^{2}},\left.\quad w\right|_{x= \pm \infty}=u_{+},
$$

has a solution $w_{0}(x)$ for some $\varepsilon_{0}>0$. Moreover, we assume that

$$
\left\|w_{0}\right\|_{\infty}<\infty, \quad\left\|\frac{\mathrm{d} w_{0}}{\mathrm{~d} x}, \frac{\mathrm{d}^{2} w_{0}}{\mathrm{~d} x^{2}}\right\|_{1}<\infty .
$$

We will comment later on the solvability of problem (4.6), (4.7). Now, we suppose that $w_{0}(x)$ exists and consider the parabolic approximation

$$
u_{t}+f(u)_{x}=\varepsilon u_{x x},\left.\quad u\right|_{t=0}=w_{0}\left(\frac{\varepsilon_{0} x}{\varepsilon}\right) \equiv u_{0}^{\varepsilon}(x) .
$$


Due to (4.7) and (4.6), the sequence $u_{0}^{\varepsilon}(x), \varepsilon \downarrow 0$, approximates the step function $u_{0}(x)$ in the sense of (1.9) and (1.10). By uniqueness, $u(t, x)$ is a travelling-wave solution and

$$
u(t, x)=w_{0}\left(\frac{\varepsilon_{0}}{\varepsilon}(x-\lambda t)\right)
$$

Now, it is clear that any function $\delta(\varepsilon)$ is a shock-layer thickness if

$$
\frac{\varepsilon}{\delta(\varepsilon)} \rightarrow 0 \quad \text { as } \varepsilon \rightarrow 0
$$

The set of fluxes $f(u)$ which guarantee solvability of problem (4.6), (4.7) is not empty. Indeed, consider the problem

$$
U_{t}+U U_{x}=0,\left.\quad U\right|_{t=0}=u_{0}(x) .
$$

As in $[\mathbf{1 2}]$, one can verify that the corresponding boundary-value problem (4.6) has the solution

$$
w=u_{-}+\frac{\left[u_{0}\right]}{1+\exp \left(\left[u_{0}\right] x / 2 \varepsilon_{0}\right)}, \quad\left[u_{0}\right]=u_{+}-u_{-},
$$

if $\lambda=\frac{1}{2}\left(u_{+}+u_{-}\right)$. This solution satisfies conditions (4.7).

Let us return to the Riemann problem (4.5) with approximation (4.8), assuming that

$$
f(u)=\lambda u+\varphi(u-\beta), \quad \beta=\frac{1}{2}\left(u_{+}+u_{-}\right), \quad \varphi^{\prime \prime}(u) \geqslant 0, \quad u_{-}>u_{+},
$$

where $\varphi$ is an even function (see (1.3) and (1.4)). Denoting

$$
z=w-\beta, \quad z_{+}=\frac{1}{2}\left(u_{+}-u_{-}\right), \quad z_{-}=-z_{+},
$$

we derive from (4.6) that

$$
\varphi(z)-\varepsilon_{0} z_{x}=c \equiv \text { const. }
$$

The condition

$$
z_{x} \rightarrow 0 \quad \text { as } x \rightarrow \pm \infty
$$

is necessary for solvability of problem (4.6), hence $c=\varphi\left(z_{+}\right)=\varphi\left(z_{-}\right)$. Due to convexity of $\varphi(u)$, it follows from (4.11) that

$$
\varphi(z)-c=\varepsilon_{0} z_{x} \leqslant 0 .
$$

Thus, any solution of (4.6) is a decreasing function, and $z_{+} \leqslant z \leqslant z_{-}$. Since $\varphi^{\prime}(0)=0$, $z_{+}<0$ and $\varphi^{\prime \prime}(z) \geqslant 0$, we have $\varphi^{\prime}\left(z_{+}\right) \leqslant 0$ and $\varphi^{\prime}\left(z_{-}\right) \geqslant 0$. Suppose that $\varphi^{\prime}\left(z_{+}\right)=0$, then necessarily

$$
\varphi^{\prime}(z)=0, \quad|z| \leqslant\left|z_{+}\right| .
$$

Now, it follows from (4.6) that

$$
z_{x x}=0, \quad x \in \mathbb{R},
$$

which contradicts the boundary conditions in (4.6). Thus, $\varphi^{\prime}\left(z_{+}\right)<0$ and $\varphi^{\prime}\left(z_{-}\right)>0$. It implies that $f^{\prime}\left(u_{-}\right)>\lambda>f^{\prime}\left(u_{+}\right)$, i.e. the shock $x=\lambda t$ is non-characteristic. 
Remark 4.3. As for the semi-characteristic shock, which is characteristic only from one side, we suppose that such a shock has the thickness $\delta(\varepsilon)$, which obeys Theorem 1.2.

Acknowledgements. The author is grateful to Professor D. Serre for stimulating discussions. The work was supported by the INTAS, under grant 01-868, the Russian Fund for Basic Research, under grant 02-01-00645, and the Russian University Programme, under grant 04.01.038.

\section{References}

1. H. Frid AND V. Shelukhin, Boundary layers for the Navier-Stokes equations of compressible fluids, Commun. Math. Phys. 208 (1999), 309-330.

2. H. Frid And V. Shelukhin, A boundary layer in parabolic perturbations of a scalar conservation law, Z. Angew. Math. Phys., in press.

3. J. Goodman and Z. XIN, Viscous limits for piecewise smooth solutions to systems of conservation laws, Arch. Ration. Mech. Analysis 121 (1992), 235-265.

4. A. M. IL'In, The boundary layers, in Encyclopedia of mathematical science, vol. 34, Partial differential equations (ed. M. V. Fedoryuk), pp. 173-210 (Springer, 1999).

5. S. N. KRUŽKOv, First order quasilinear equations in several independent variables, Mat. USSR Sb. 10 (1970), 217-243.

6. L. D. Landau and E. M. Lifshitz, Fluid mechanics, 2nd edn (Pergamon Press, Oxford, 1987).

7. J. L. LiOnS AND E. MAGENIS, Non-homogeneous boundary value problems and applications, vol. II (Springer, 1972).

8. T. P. LiU, Nonlinear stability of shock waves for viscous conservation laws, Memoirs of the American Mathematical Society, vol. 328 (1985).

9. J. MALEC, J. NeC̆AS, M. ROKYTA AND M. RUŽIC̆KA, Weak and measure-valued solutions to evolutionary PDEs (Chapman \& Hall, London, 1996).

10. R. Mises, Mathematical theory of compressible fluid flow (Academic, 1958).

11. V. V. OstapenKo, On convergence of through finite difference schemes, invariant with respect to the similarity transform, at shocks, J. Computat. Math. Math. Phys. 26 (1986), 1661-1678 (in Russian).

12. B. L. RozDeStVEnSKiI AND N. N. YANEnKo, Systems of quasilinear equations and their applications to gas dynamics, AMS Translations of Mathematical Monographs, vol. 55 (American Mathematical Society, Providence, RI, 1983).

13. D. Serre, Systèmes de lois de conservation, vols I and II (Diderot, Art et Sciences, Paris, 1996).

14. D. Serre And K. Zumbrun, Boundary layer stability in real vanishing viscosity limit, Commun. Math. Phys. 221 (2001), 267-292. 